\title{
COMPARISON BETWEEN ELECTROCHROMIC AND PHOTOCHROMIC COLORATION EFFICIENCY OF TUNGSTEN OXIDE THIN FILMS
}

\author{
C. Bechinger, ${ }^{a}$ M.S. Burdis ${ }^{b}$ and J.-G. Zhang ${ }^{c, *}$ \\ ${ }^{a}$ Universitaet Konstanz, Postfach 5560 M675, D-78434 Konstanz, Germany \\ ${ }^{b}$ Pilkington Tech. Management Ltd., Hall Lane Lathom, Ormskirk L40 5UF, Lancashire, England \\ 'National Renewable Energy Laboratory, 1617 Cole Boulevard, Golden, CO 80401, U.S.A.
}

\begin{abstract}
We investigated the photochromic (PC) and electrochromic (EC) behavior of sputtered tungsten oxide $\left(\mathrm{WO}_{3-y}\right)$ films with different oxygen deficiency $y$. It was found that both the PC and EC coloration efficiency increase with increasing oxygen deficiency in tungsten oxide. For PC efficiency, this behavior is consistent with the model of photochromism developed recently. A recently developed model based on the small polaron transition between $\mathrm{W}^{5+}$ and $\mathrm{W}^{4+}$ states has been used to explain the dependence of EC coloring efficiency on the oxygen deficiency in tungsten oxide films. This new mechanism also revealed a close relationship between PC and EC. (O) 1997 Published by Elsevier Science Ltd. All rights reserved
\end{abstract}

Keywords: A. thin films, D. optical properties, E. light absorption and reflection.

Thin films of transition metal oxides have many interesting properties. One of their most important features is that their optical absorption can be switched reversibly under certain conditions. This chromogenic behavior is displayed most prominently in thin films of amorphous tungsten oxide $\left(\mathrm{WO}_{3-y}\right)$, the most promising material for "smart windows" [1] and large area passive displays [2]. Coloration and bleaching can be accomplished in different ways. Electrochromic (EC) coloration, e.g. is obtained when ions and electrons are injected electrochemically from an external source into a $\mathrm{WO}_{3-y}$ layer by means of a small voltage. Upon reversing this voltage, the device can be bleached again. This behavior is usually explained in terms of the double-charge-injection (DCI) model [3] that describes the formation of a tungsten bronze $\left(\mathrm{M}_{x} \mathrm{WO}_{3-y}\right)$ with injection of electrons and positively charged ions $\left(\mathrm{M}=\mathrm{H}^{+}, \mathrm{Li}^{+}, \mathrm{Na}^{+}\right.$, etc.) into the transparent $\mathrm{WO}_{3}$ :

$$
\underset{\text { (transparent) }}{\mathrm{WO}_{3-y}+x \mathrm{e}^{-}+x \mathrm{M}^{+} \Leftrightarrow \mathrm{M}_{x} \mathrm{WO}_{3-y} .}
$$

- To whom all correspondence should be sent.
During charge injection, electrons are localized at tungsten sites thereby changing the valence of the tungsten ions from $6^{+}$to $5^{+}$. These localized electrons accompany a lattice distortion that forms small polarons [4]. Under the absorption of light these small polarons can hop from sites A to B. This process which is responsible for the color in tungsten oxide is referred to as intervalence transfer and can be expressed as

$h \nu+\mathrm{W}^{5+}(\mathrm{A})+\mathrm{W}^{6+}(\mathrm{B}) \Rightarrow \mathrm{W}^{5+}(\mathrm{B})+\mathrm{W}^{6+}(\mathrm{A})$.

Although this model is widely accepted, it still cannot explain some key experimental results. For example, in contrast to what one would expect, nuclear reaction analysis (NRA) experiments could not provide proof of a systematically increased hydrogen content of the colored films when protons were used as the injected ions $[5,6]$. Furthermore, the DCI model accounts only for EC but not for PC coloration, where the same absorption band is generated simply by irradiation of a bare $\mathrm{WO}_{3-y}$ layer with ultraviolet (UV) light [7]. Although (in the case of PC) an external injection of charges can be ruled out, electrical $[8,9]$, structural $[10]$ and other studies $[11,12]$ show that the properties of 
color centers in tungsten oxide are very similar whether they are formed by PC or EC coloration. These findings raise the question as to whether there is a more generalized model that can account for both coloration processes.

The aim of this paper is to discuss a new important feature that reveals the close relationship between EC and PC effects. We found a very similar dependence of the EC and PC coloration efficiency on the oxygen stoichiometry $y$ of sputtered $\mathrm{WO}_{3-y}$ samples. A new mechanism for coloration (being consistent with earlier experiments) is proposed that explains the results in this work.

Thin $\mathrm{WO}_{3}$ films were concurrently deposited onto glass and ITO-coated glass substrates. This allowed the characterization of both the EC and PC effects, as well as the measurement of the optical characteristics. The deposition system was a radio frequency (RF) diode sputterer with a $15-\mathrm{cm}$ diameter target. Two mass-flow controllers were used to control the sputtering atmosphere, and a throttled diffusion pump allowed control of the total pressure. The total pressure was $1.6 \times 10^{-2} \mathrm{mbar}$, the total RF power was $200 \mathrm{~W}$ at $13.56 \mathrm{MHz}$. All conditions were maintained constant except for $\mathrm{O}_{2}$ concentration in argon, which was varied between $5 \%$ and $50 \%$. Consistent with other authors $[13,14]$ we found that the deposition rate varied only slightly when the oxygen partial pressure was varied between 5 and $50 \%$. Therefore, only small variations of the deposition time were needed to obtain films with approximately the same thickness $(3000 \AA)$. There was no intentional substrate heating during film deposition and the resulting films were amorphous as measured by $\mathrm{X}$-ray diffraction. The oxygen deficiency in all our samples was determined by XPS analysis. Before each measurement a surface layer of about $15 \AA$ was removed with a $1 \mathrm{kV}$ argon sputter beam, in order to eliminate surface contamination of our samples with carbon. We did not calibrate the $\mathrm{W}$ and $\mathrm{O}$ cross sections to a $\mathrm{WO}_{3}$ standard because we are only interested in relative $\mathrm{O} / \mathrm{W}$ ratios rather than absolute values. The samples sputtered in the same run were used in both PC and EC efficiency measurement, so we can largely exclude differences introduced during sample preparation.

The PC coloration was induced with a $75 \mathrm{~W}$ xenon high-pressure lamp. A water filter was used to minimize the thermal effects. The incident UV intensity on the sample was about $250 \mathrm{~mW} \mathrm{~cm}^{-2}$. The samples were exposed to UV light of the same intensity for $15 \mathrm{~min}$, which caused a decrease in transmission of typically a few percentage points. The PC response of the samples was probed with the transmission of an incoherent light beam at a wavelength of $700 \pm 15 \mathrm{~nm}$. The change of the optical density, $\Delta O . D$., which is proportional to the amount of created color centers, was calculated by the equation

$\Delta$ O.D. $=\log _{10}\left(\frac{T_{b}}{T_{c}}\right)$,

where $T_{b}$ and $T_{c}$ are the transmittances before and after coloration, respectively. We can define the PC efficiency as $\eta_{\mathrm{PC}}=\Delta \mathrm{O} . \mathrm{D} . / \mathrm{It}$, where $I$ is the light intensity and $t$ is the exposure time. Since a constant light intensity $I$ and time $t$ was used through this work, $\eta_{\mathrm{PC}}$ is directly proportional to $\triangle O . D$.

To determine the EC efficiency, the samples were colored in an electrochemical cell. A Schlumberger 1286 electrochemical interface (ECI) was used to control the potential of the $\mathrm{WO}_{3}$ working electrode relative to a lithium metal reference electrode. Lithium metal was also used as the counter electrode. The electrolyte used was a carefully dried solution of $0.5 \mathrm{M}$ lithium trifiate in propylene carbonate. We used the galvanostatic mode of the ECI to allow accurate measurement of the amount of charge $\Delta Q=20 \mathrm{mC}$ inserted into the $\mathrm{WO}_{3}$ under constant current. The sample transmittance was measured before and after charge injection $\Delta O . D$. was obtained according to equation (3). To calculate the EC efficiency $\eta_{\mathrm{EC}}$, we used the ratio of the change in optical density and the amount of injected charge per unit sample area $\eta_{\mathrm{EC}}=\Delta \mathrm{O} . \mathrm{D} . / \Delta Q$.

Figure 1 shows that the $\mathrm{O} / \mathrm{W}$ ratio of our $\mathrm{WO}_{3}$ samples increases with increasing oxygen partial pressure in the sputtering gas. In terms of oxygen deficiency, $y$, this means that $y$ decreases with increasing $\mathrm{O}_{2}$ pressure. This result is in agreement with other authors who found that the $y$ values in $\mathrm{WO}_{3-y}$ can be varied between 0 and 0.5 depending on the oxygen partial pressure. Deneuville et al. suggested that stoichiometric

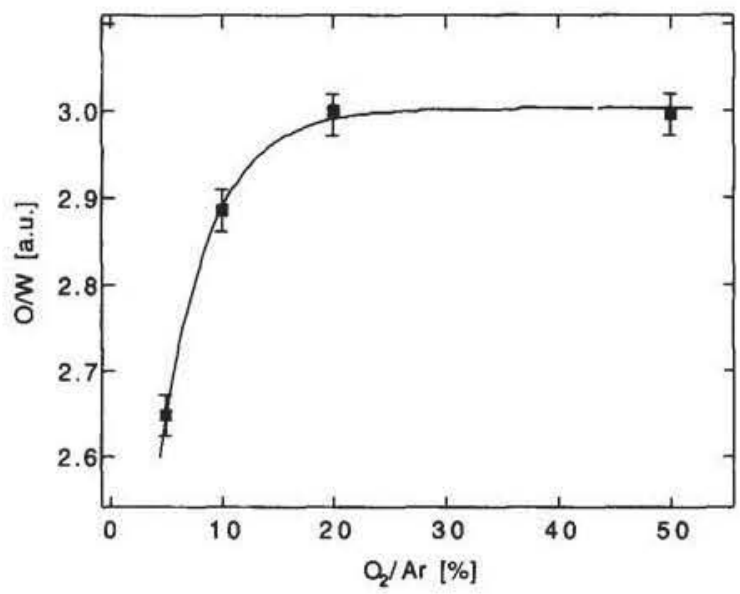

Fig. 1. $\mathrm{O} / \mathrm{W}$ ratio of sputtered $\mathrm{WO}_{3-y}$ films determined with XPS measurements as a function of the $\mathrm{O}_{2}$ concentration in the sputtering gas. The sputter target was $\mathrm{WO}_{3}$. The line is a guide to the eye. 


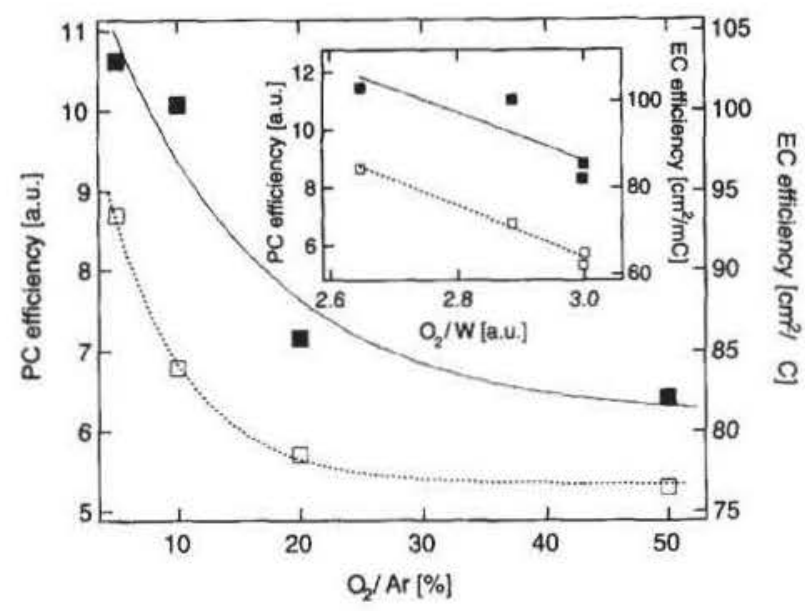

Fig. 2. Coloration efficiencies of sputtered tungsten oxide films as a function of the $\mathrm{O}_{2}$ concentration in the sputtering gas. Open and closed symbols denote the PC and EC coloration efficiency, respectively. The inset shows the PC and EC efficiency as a function of the $\mathrm{O} / \mathrm{W}$ ratio, which has been taken from Fig. 1. The lines are guides to the eye.

samples will only be obtained if $\mathrm{WO}_{3}$ is sputtered in a pure oxygen atmosphere [15]. On the other hand, $\mathrm{WO}_{2.5}$ films are obtained when the sputter gas contains only a very small amount of oxygen [14]. Several groups also reported that $\mathrm{WO}_{3}$ samples may have essentially the same optical absorption but large variation in their oxygen deficiency $[5,16]$. The optical transmission spectra of the samples used in this work were almost identical, but these films have different oxygen deficiencies which depend on the different oxygen partial pressures used during the film deposition. The open symbols in Fig. 2 show the results of the PC efficiency as a function of the oxygen gas content in the sputtering gas and the O/W ratio (see inset), respectively. We observed a drop of the coloration efficiency by about $40 \%$ when the oxygen content in the sputtering gas increases from $5 \%$ to $50 \%$. Since the oxygen deficiency, $y$ (i.e. the number of oxygen vacancies), decreases with increasing oxygen content, this shows that the existence of oxygen vacancies is crucial for the PC response.

The decrease of the PC efficiency with decreasing $y$ is consistent with a model developed recently for photochromism in tungsten oxide $[17,18]$. It has been suggested that the PC effect in $\mathrm{WO}_{3-y}$ is intimately connected to the photoinduced decomposition of incorporated water, the latter being well known to be present in amorphous tungsten oxide films. The formation of color centers is also accompanied by the formation of highly reactive oxygen radicals. To prevent the instantaneous back-reaction of these O-radicals back to water (which would hinder the formation of a color center), it is important to separate the oxygen radicals immediately. There are two separation mechanisms that have to be considered: the first one (occurring only in samples considerably thinner than those discussed here) is the escape of free oxygen out of the sample during light exposure. The second possibly (the essential one for the understanding of the results discussed in this paper) is that $\mathrm{O}$-atoms temporarily occupy oxygen vacancies. As the number of oxygen vacancies decreases with increasing oxygen partial pressure during sputtering, the separation of oxygen from the reaction center after its release from photoinduced splitting of water is getting more and more difficult. This eventually leads to a suppressed PC coloration efficiency as shown in Fig. 2. Accordingly, we expect for nearly fully oxidized tungsten oxide films, i.e. $y \approx 0$, a largely suppressed PC coloration efficiency. Indeed, this has been observed by measurements of Gerard and Deneuville [5].

The coloration efficiency for the EC effect is shown in Fig. 2 as solid symbols. Similar to the PC efficiency, the EC efficiency decreases with increasing oxygen partial pressure in the sputtering gas, i.e. with decreasing oxygen deficiency $y$ (see inset). This behavior, which has also been observed by Kitao et al. [14] can not be explained by the double charge injection model. Here we propose a new scheme to explain the experimental results regarding the EC efficiency (a more detailed description will be published elsewhere [19]). Desorption studies of tungsten oxide surfaces indicate that $\mathrm{O}$, WO, $\mathrm{WO}_{2}, \mathrm{WO}_{3}, \mathrm{~W}_{2} \mathrm{O}_{6}$ and $\mathrm{W}_{3} \mathrm{O}_{9}$ are desorbed when the material is heated [20-22]. In contrast, moieties like $\mathrm{W}_{2} \mathrm{O}_{5}$ are not observed. This indicates that $\mathrm{W}^{6+}$ and $\mathrm{W}^{4+}$ states are much more easy to form than $\mathrm{W}^{5+}$ states during a physical deposition/desorption process. Therefore, the tungsten in virgin tungsten oxide samples may be considered mainly as a mixture of mainly $\mathrm{W}^{6+}$ and $\mathrm{W}^{4+}$ states. Accordingly, we expect an increase of $\mathrm{W}^{4+}$ states with increasing oxygen deficiency of the samples. This has been confirmed by Gerard et al. with XPS measurements [23].

After EC coloration [cf. equation (1)] the number of $\mathrm{W}^{5+}$ states increases with increasing injected charge. However, in contrast to the usually assumed coloration mechanism, i.e. the intervalence transfer between $\mathrm{W}^{5+}$ to $\mathrm{W}^{6+}$ states, here we propose a transition between $\mathrm{W}^{5+}$ to $\mathrm{W}^{4+}$ states. Obviously, the strength of the absorption depends on the concentrations of these two valency states in the samples. Because the number of $\mathrm{W}^{4+}$ states increases with increasing oxygen deficiency in the virgin samples, EC efficiency will also increase with increasing oxygen deficiency of the samples (up to a certain limit, e.g. $y<0.3$ ). A very similar idea, i.e. an intervalence transfer between $\mathrm{W}^{5+}$ and $\mathrm{W}^{4+}$ states, can also be applied to photochromism. This does not change the arguments we used above to describe the dependence 
of PC efficiency on the oxygen content of the sample. In fact, it enhances the importance of the oxygen deficiency during PC coloration, because now the oxygen deficiency (in addition to the arguments above) is also responsible for $\mathrm{W}^{4+}$ formation that additionally increases PC efficiency.

In summary, we investigated the coloration efficiency of sputtered $\mathrm{WO}_{3-y}$ films with different $y$. Variation of $y$ was generated by changing the $\mathrm{O}_{2}$ content in the sputtering gas from $5 \%$ to $50 \%$ and confirmed with XPS measurements. Both photochromism and electrochromism of these samples were found to show an increase of coloration efficiency with increasing oxygen deficiency $y$. The results for the PC efficiency have been explained in terms of a recently developed model but the results for EC efficiency can not be explained by the conventional double-charge-injection model. We suggest a new model where intervalence transfer takes place between $\mathrm{W}^{5+}$ and $\mathrm{W}^{4+}$ states. This model can explain the experimental results on the EC efficiency. It also supports the importance of the oxygen deficiency for PC efficiency.

Acknowledgements-We gratefully acknowledge the help of David Niles (NREL) for doing XPS measurements on our samples and D.K. Benson (NREL) for helpful discussions. One of us (C.B.) is supported by the U.S. Department of Energy, Advanced Energy Project, Office of Basic Energy Sciences and Deutsche Forschungsgemeinschaft.

\section{REFERENCES}

1. Lampert, C.M., Sol. En. Mat. Sol. Cells, 32, 1994, 307.

2. Donnadieu, A., Mater. Sci. Eng., B3, 1989, 185.

3. Crandall, R.S. and Faughnan, B.W., Appl. Phys. Lett., 26, 1975, 120.

4. Schirmer, O.F., Wittwer, V., Baur, G. and Brand, G., J. Electrochem. Soc., 124, 1977, 749.
5. Gerard, P., Deneuville, A. and Courths, R., Thin Solid Films, 71, 1980, 221.

6. Wagner, W., Rauch, F., Ottermann, C. and Bange, K., Nuclear Instr. Methods Phys. Res., B50, 1990, 27.

7. Deb, S.K., Philos. Mag., 27, 1973, 801.

8. Crandall, R.S. and Faughnan, B.W., Phys. Rev. Lett., 39, 1977, 232.

9. Bechinger, C., Herminghaus, S. and Leiderer, P., Thin Solid Films, 239, 1994, 156.

10. Gavrilyuk, A.I., Prokhvatilov, V.G. and Chundnovskii, F.A., Sov. Phys. Solid State, 24, 1982, 558.

11. Gazzinelli, R. and Schirmer, O.F., J. Phys., C10, 1977, L145.

12. Temmink, A., Anderson, O., Bange, K., Hantsche, H. and Yu, X., Thin Solid Films, 192, 1990, 211.

13. Akram, H., Kitao, M. and Yamada, S., J. Appl. Phys., 66, 1989, 4364.

14. Kitao, M., Yamada, S., Yoshida, S., Akram, H. and Urabe, K., Sol. En. Mat. Sol. Cells, 25, 1992, 241.

15. Deneuville, A. and Gerard, P., J. Electron. Mat., 7, 1978, 559.

16. Demiryont, H. and Schulz, S.C., Optical Materials Technology for Energy Efficiency and Solar Energy Conversion VIII (Edited by C.G. Granqvist and C.M. Lampert), SPIE 1149, 1989, 28.

17. Bechlinger, C., Oefinger, G., Herminghaus, S. and Leiderer, P., J. Appl. Phys., 74, 1993, 4527.

18. Bechinger, C., Ebner, D., Herminghaus, S. and Leiderer, P., Solid State Commun., 89, 1994, 205.

19. Zhang, J.-G., Benson, D.K., Tracy, C.E., Deb, S.K., Czanderna, A.W. and Bechinger, C. To be published in J. Electrochem. Soc.

20. Ageev, V.N. and Ionov, N.I., Sov. Phys. Tech. Phys., 10, 1966, 1614.

21. King, D.A., Madey, T.E. and Yates, J.T., J. Chem. Phys., 55, 1971, 3236.

22. Granqvist, C.G., Handbook of Inorganic Electrochromic Materials, p. 31. Elsevier, New York, 1995.

23. Gerard, P., Deneuville, A., Hollinger, G. and Duc, T.M., J. Appl. Phys., 48, 1977, 4252. 